\title{
Numerical calculation of heat and mass transfer processes in the adsorbers of the air purification system
}

\author{
O.V. Tushavina1 ${ }^{1}$ E.R. Sadretdinova ${ }^{2}$ \\ ${ }^{1,2}$ Moscow Aviation Institute (National Research University), Volokolamskoeshosse, 4, 125993, Moscow, Russia \\ solgtu@gmail.com
}

Article History: Received: 11 January 2021; Accepted: 27 February 2021; Published online: 5 April 2021

Abstract:In this work, a series of parametric calculations was made for the processes of sorption and desorption of water vapor in relation to the conditions of the process of sorption air purification. A mathematical model was developed that adequately reflects the processes of heat and mass transfer in the adsorption unit at all stages of the adsorption cycle. Algorithms for solving problems and programs for calculating heat and mass transfer processes in an adsorption regenerated installation are obtained, results of parametric calculations of heat and mass transfer processes at each stage of the adsorption cycle and for the entire cycle as a whole are obtained.

Keywords: air purification, adsorption, heat and mass transfer, mathematical model

\section{Introduction}

The main objective of research to improve adsorption air purification systems is to reduce energy costs. In addition, as long operating experience has shown, the urgent task is to create an automatic control system, which is necessary to control and optimize the operating modes of the installation. In most cyclic adsorption plants, the issue of increasing the output characteristics is also relevant. In particular, one of the requirements for the predrying unit is to ensure, as possible, a lower dew point temperature of the air at the outlet from the unit (not higher than minus $40 \mathrm{C}$ ) over the entire range of operating parameters of the system. This requirement is primarily associated with the need to carry out more efficient air purification from carbon dioxide.

The solution of the set tasks requires a large amount of theoretical and experimental work [1-7]. Due to the large number of parameters that determine the efficiency of the drying process, a complete cycle analysis is largely determined by the availability and implementation of reliable mathematical models and calculation algorithms in engineering practice. On their basis, it is possible both to take into account the features of the flow of heat and mass transfer processes at each of the steps of the cycle, and their interaction during the transition from the previous step to the next [7-14]. Numerical experiments on predicting the behavior of an adsorption system with different organization of cycle steps make it possible not only to reasonably determine the technological parameters of the process, but in many cases also to reduce the amount of experimental research.

Adsorption is a very widely extended physical process, involving creation of mono- or polymolecular layer on the surface [15-27]. A huge amount of studies apply adsorption for creation of hybrid materials [28-42]. In the course of operation of adsorbers in absorption cartridges, a complex interaction of mass and heat transfer processes occurs. Mass transfer is determined by the sorption-desorption of water vapor when moist air is filtered through the absorber layer. Sorption processes are accompanied by significant thermal effects. On the other hand, the temperature of the sorbent granules strongly affects their sorption capacity. Thus, the interaction of heat and mass flows in the sorbent arises, on which the effect of convective transfer is superimposed during gas filtration through the absorber.

As a result of sorption of water vapor, heat is released, the specific value of which per unit mass of the sorbent is comparable in order to the heat of condensation of water. On the contrary, during desorption, energy is absorbed.

\section{Modeling heat and mass transfer in adsorption processes}

For the numerical solution of heat and mass transfer problems, the system of differential equations is replaced by a system of difference equations. Integration of the system of equations using conventional numerical methods leads to an excessively large amount of computations, since it is necessary to simultaneously integrate all $2 \mathrm{~N}$ (for some fixed value of $\mathrm{N}$ ) equations at once, while moving one step in time. To reduce computational costs, in this case, a numerical method of splitting into physical processes is used. The essence of this method lies in the fact that various equations of the system are solved numerically one by one at each time step using finite difference methods. 
In this case, the system is integrated in the following sequence. First, the mass transfer equations for all $(N-1)$ components are integrated. When moving to the next time layer, the densities of each $i$-th $(i=1 \div(N-1)$ ) component are determined by their values on the previous time layer. The values of the convective transfer rate $U$ and the velocities of the mass $\partial a_{i} / \partial t$ are not determined for the next time layer while. They remain fixed for now. Next, the energy equation is integrated, from which the temperature is determined at each point (node) of the grid of the computational domain on the next one, i.e. in the same place as in the integration of the mass transfer equation for the time layer. In this case, the value of the density $\rho$, velocity, $U$, and quantities $a_{i}$, required to solve the energy equation are taken from the initial time layer. Themselves, these quantities $\rho, U, a_{i}$ do not change when solving the energy equation. From the algebraic equation of state for the found new temperature value, a new value of the average gas density is determined $\rho$. Then, using both the new and the old value $\rho$, the convective transfer rate is determined $U$. The latter determine the values using equations $a_{i}$ which are solved by the usual Runge-Kutta method, for each node of the computational grid separately.

This completes the calculation of all the required quantities in one time step. The sequence of integration of equations in the method of splitting into processes can be arbitrary. The final solution of the system should not depend on the order in which the equations are integrated.

The numerical methods and software were tested by comparing the calculation results with the exact analytical solutions obtained under some simplifying assumptions. In addition, the fulfillment of the integral relation describing the mass balance over a finite period of time for the entire layer as a whole was checked.

An analytical solution to the transport equation for one component can be obtained by assuming $\varepsilon, U$, and $\rho$ are constant and the derivative $\frac{\partial a_{i}}{\partial t}=\beta\left(\rho-\rho^{*}\right)$, then

$$
\varepsilon \frac{\partial \rho}{\partial t}+U \frac{\partial \rho}{\partial x}=-\beta\left(\rho-\rho^{*}\right)
$$

Quasilinear first-order partial differential equation general solution of the form $F\left(C_{1} ; C_{2}\right)=0$, whereF - is an arbitrary function, and $C_{1}(t, x)$ and $C_{2}(t, x)$ are functionally independent first integrals of the characteristic system

$$
\frac{\partial t}{\varepsilon}=\frac{\partial x}{U}=\frac{\partial \rho}{\rho-\rho^{*}} .
$$

The first integrals of the system will be

$$
\begin{aligned}
& C_{1}=t-\frac{\varepsilon}{U} x \\
& C_{2}=\ln \left|\rho-\rho^{*}\right|+\frac{\beta t}{\varepsilon} .
\end{aligned}
$$

To determine the specific type of function, it is necessary to set the boundary and initial conditions. Initialconditions:

$$
t=0, \quad \rho=\rho_{0}(x)
$$

Let us set this condition in parametric form

$$
t=0, \quad x=\xi, \quad \rho=\rho_{0}(\xi)
$$

where $\xi$ - parameter. Substituting condition (4) into system (2), (3) and excluding the parameter $\xi$ from it,

we have: $\quad \ln \left|\rho_{0}\left(-\frac{U}{\varepsilon} C_{1}\right)-\rho^{*}\right|=C_{2}$

Substituting now expressions (3), (4) into (6), a solution is obtained that satisfies the initial condition (5):

$$
\rho(t, x)=\rho^{*}+\left[\rho_{0}\left(x-\frac{U}{\varepsilon} t\right)-\rho^{*}\right] e^{-\frac{\beta t}{\varepsilon}} \text {. }
$$

Here, as before, the expression in parentheses after $\rho_{0}$ is the function argument $\rho_{0}(\xi)$. 
We also set theboundary conditionsin the parametric form:

$$
x=0, \quad t=\xi, \quad \rho=\rho_{1}(\xi)
$$

where $\xi$ - parameter, and $\rho_{1}(\xi)=\rho_{1}(t)$ - a function describing changes in concentration over time at the entrance to the layer. Following the same procedure for excluding a parameter using expressions for the first integrals (3), (4), a solution is obtained in the following form:

$$
\rho(t, x)=\rho^{*}+\left[\rho_{1}\left(t-\frac{\varepsilon}{U} x\right)-\rho^{*}\right] e^{-\frac{\beta x}{U}}
$$

with the simultaneous setting of the initial and boundary conditions, the solution consists of two parts, each of which is determined by relations (7) or (9) depending on which side of the front of the flow moving with speed and separating the regions of the initial and boundary conditions, the considered section is found.

$$
\begin{aligned}
& \text { When } t<\frac{\varepsilon}{U} x: \rho(t, x)-\text { calculated by (7) } \\
& \text { When } t>\frac{\varepsilon}{U} x: \rho(t, x) \text { - calculated by (9) }
\end{aligned}
$$

To test the program for solving the transport equation, the following initial and boundary conditions were set:

$$
t=0, \quad \rho_{0}(x)=0, \quad x=0, \quad \rho_{1}(t)=A \cdot \sin ^{2}\left(\frac{2 \pi}{T_{1 / 2}} \cdot t\right),
$$

where $A$ - the amplitude of the input concentration fluctuations, $T_{1 / 2}$ its half-period. Comparison of numerical calculations with analytical ones obtained by formulas (7), (9) in accordance with (10) showed satisfactory agreement.

Another way to test the program: checking the fulfillment of the mass conservation law written in integral form for the layer as a whole.

$$
\varepsilon \frac{\partial \rho}{\partial t}+\frac{\partial(U \rho)}{\partial x}+\frac{\partial a}{\partial t}=0
$$

Integrating this equation over the coordinate from $x_{1}$ to $x_{2}$ and in time from 0 to $t$, we get:

$$
F \int_{x_{1}}^{x_{2}}(\varepsilon \rho+a) d x+\int_{0}^{t} F U_{2} \rho_{2} d t^{\prime}-\int_{0}^{t} F U_{1} \rho_{1} d t^{\prime}=0
$$

where $\quad x_{1}, U_{1}, \rho_{1}$ - values at the entrance to the layer, $U_{2}, \rho_{2}-$ at the exit from the layer.

Relation (11) represents the balance of mass: the amount of a substance in a layer in a free and bound state is equal to the difference in the masses of a substance supplied to the layer and exited from it along with the air flow.

\section{Conclusions}

The use of the obtained expressions to control the operation of the program has a significant advantage in comparison with the previously considered method: the program can be checked in the process of solving the problem and in the most general setting without idealizing the problem.Thus, the studies carried out have confirmed the correctness of the physical and mathematical model, which makes it possible to use it in the future to analyze the process of sorption air purification.

\section{Acknowledgements}

This work was carried out with the financial support of RFBR, project No. 20-08-00880

\section{References}

1. Rabinsky, L.N., Kuznetsova, E.L. Simulation of residual thermal stresses in high-porous fibrous silicon nitride ceramics // Powder Metallurgy and Metal Ceramics, 2019, 57(11-12), p. 663-669.

2. Rabinskiy, L.N. Non-stationary problem of the plane oblique pressure wave diffraction on thin shell in the shape of parabolic cylinder// PeriodicoTcheQuimica, 2019, 16(32), p. 328-337. 
3. Formalev, V.F., Kolesnik, S.A., Selin, I.A. Local non-equilibrium heat transfer in an anisotropic halfspace affected by a non-steady state point heat source // Herald of the Bauman Moscow State Technical University, Series Natural Sciences. 2018. 80(5), p. 99-111.

4. Kolesnik, S.A., Bulychev, N.A., Rabinskiy, L.N., Kazaryan, M.A. Mathematical modeling and experimental studies of thermal protection of composite materials under high-intensity effects of laser radiation// Proceedings of SPIE - The International Society for Optical Engineering. 2019. 11322, article number $113221 \mathrm{R}$.

5. Kuznetsova, E.L., Rabinskiy, L.N. Heat transfer in nonlinear anisotropic growing bodies based on analytical solution // Asia Life Sciences, 2019, (2), p. 837-846.

6. Kuznetsova, E.L., Rabinskiy, L.N. Numerical modeling and software for determining the static and linkage parameters of growing bodies in the process of non-stationary additive heat and mass transfer//PeriodicoTcheQuimica, 2019, 16(33), p. 472-479.

7. Kuznetsova, E.L., Rabinskiy, L.N. Linearization of radiant heat fluxes in the mathematical modeling of growing bodies by the action of high temperatures in additive manufacturing //Asia Life Sciences, 2019, (2), p. 943-954.

8. Antufev, B.A., Egorova, O.V., Rabinskiy, L.N. Quasi-static stability of a ribbed shell interacting with moving load// INCAS Bulletin, 2019, 11, p. 33-39.

9. Bodryshev, V.V., Babaytsev, A.V., Rabinskiy, L.N. Investigation of processes of deformation of plastic materials with the help of digital image processing// PeriodicoTcheQuimica, 2019, 16(33), p. 865-876.

10. Formalev, V.F., Kartashov, É.M., Kolesnik, S.A. On the Dynamics of Motion and Reflection of Temperature Solitons in Wave Heat Transfer in Limited Regions // Journal of Engineering Physics and Thermophysics, 2020, 93(1), p. 10-15.

11. Babaytsev, A.V., Orekhov, A.A., Rabinskiy, L.N. Properties and microstructure of AlSi10Mg samples obtained by selective laser melting// Nanoscience and Technology: An International Journal, 2020, 11(3), p. 213-222.

12. Egorova, O.V., Kyaw, Y.K. Solution of inverse non-stationary boundary value problems of diffraction of plane pressure wave on convex surfaces based on analytical solution//Journal of Applied Engineering Science, 2020, 18(4), p. 676-680.

13. Formalev, V.F., Bulychev, N.A., Kuznetsova, E.L., Kolesnik, S.A. The Thermal State of a Packet of Cooled Microrocket Gas-Dynamic Lasers // Technical Physics Letters, 2020, 46(3), p. 245-248.

14. Astapov, A.N., Kuznetsova, E.L., Rabinskiy, L.N. Operating capacity of anti-oxidizing coating in hypersonic flows of air plasma//Surface Review and Letters, 2019, 26(2), 1850145 p.

15. A.N. Tarasova. Vibration-based Method for Mechanochemical Coating Metallic Surfaces, International Journal of Pharmaceutical Research, 2020, Vol. 12, Supplementary Issue 2, pp. 1160-1168.

16. Bulychev N. A., Kuznetsova E.L., Bodryshev V. V.Rabinskiy L.N. Nanotechnological aspects of temperature-dependent decomposition of polymer solutions, Nanoscience and Technology: An International Journal, 2018, Vol. 9 (2), p.91-97.

17. Bulychev, N.A., Rabinskiy, L.N. Ceramic nanostructures obtained by acoustoplasma technique//Nanoscience and Technology: An International Journal, 2019, 10(3), p. 279-286.

18. Anikin V.A., Vyshinsky V.V., Pashkov O.A., et al. Using the maximum pressure principle for verification of calculation of stationary subsonic flow. Herald of the Bauman Moscow State Technical University, Series Mechanical Engineering, 2019, no. 6, pp. 4-16.

19. A.N. Tarasova. Effect of Reagent Concentrations on Equilibria in Water-Soluble Complexes, International Journal of Pharmaceutical Research, 2020, Vol. 12, Supplementary Issue 2, pp. 11691172.

20. Yu.V. Ioni, A. Ethiraj. New Tailor-Made Polymer Stabilizers for Aqueous Dispersions of Hydrophobic Carbon Nanoparticles, International Journal of Pharmaceutical Research, 2020, Vol. 12, Issue 4, pp. 3443-3446.

21. Yu.V. Ioni. Nanoparticles of noble metals on the surface of graphene flakes, PeriodicoTcheQuimica, 2020, Vol. 17, No. 36, pp. 1199-1211.

22. N.A. Bulychev, M.A. Kazaryan. Optical Properties of Zinc Oxide Nanoparticles Synthesized in Plasma Discharge in Liquid under Ultrasonic Cavitation, Proceedings of SPIE, 2019, Vol. 11322, article number 1132219.

23. A.N. Tarasova. Effect of Vibration on Physical Properties of Polymeric Latexes, International Journal of Pharmaceutical Research, 2020, Vol. 12, Supplementary Issue 2, pp. 1173-1180.

24. B.A. Garibyan. Mechanical Properties of Electroconductive Ceramics, International Journal of Pharmaceutical Research, 2020, Vol. 12, Supplementary Issue 2, pp. 1825-1828.

25. N.A. Bulychev, A.V. Ivanov. Effect of vibration on structure and properties of polymeric membranes, International Journal of Nanotechnology, 2019, Vol. 16, Nos. 6/7/8/9/10, pp. $334-343$. 
26. B.A. Garibyan. Enhancement of Mechanical Properties of Inorganic Glass under Ultrasonic Treatment, International Journal of Pharmaceutical Research, 2020, Vol. 12, Supplementary Issue 2, pp. 18291832.

27. B.A. Garibyan. Modelling of Technical Parameters of Discharge Reactor for Polymer Treatment, International Journal of Pharmaceutical Research, 2020, Vol. 12, Supplementary Issue 2, pp. 1833 1837.

28. M.O. Kaptakov. Effect of Ultrasonic Treatment on Stability of TiO2 Aqueous Dispersions in Presence of Water-Soluble Polymers, International Journal of Pharmaceutical Research, 2020, Vol. 12, Supplementary Issue 2, pp. 1821-1824.

29. O.A. Butusova. Surface Modification of Titanium Dioxide Microparticles Under Ultrasonic Treatment, International Journal of Pharmaceutical Research, 2020, Vol. 12, I. 4, pp. 2292-2296.

30. M.O. Kaptakov. Catalytic Desulfuration of Oil Products under Ultrasonic Treatment, International Journal of Pharmaceutical Research, 2020, Vol. 12, Supplementary Issue 2, pp. 1838-1843.

31. N.A. Bulychev, A.V. Ivanov. Nanostructure of Organic-Inorganic Composite Materials Based on Polymer Hydrogels, International Journal of Nanotechnology, 2019, Vol. 16, Nos. 6/7/8/9/10, pp. 344 355 .

32. M.O. Kaptakov. Enhancement of Quality of Oil Products under Ultrasonic Treatment, International Journal of Pharmaceutical Research, 2020, Vol. 12, Supplementary Issue 2, pp. 1851-1855.

33. Yu.V. Ioni. Synthesis of Metal Oxide Nanoparticles and Formation of Nanostructured Layers on Surfaces under Ultrasonic Vibrations, International Journal of Pharmaceutical Research, 2020, Vol. 12, Issue 4, pp. 3432-3435.

34. Yu.V. Ioni, A. Ethiraj. Study of Microparticles Surface Modification by Electrokinetic Potential Measuring, International Journal of Pharmaceutical Research, 2020, Vol. 12, Issue 4, pp. 3436-3439.

35. O.A. Butusova. Stabilization of Carbon Microparticles by High-Molecular Surfactants, International Journal of Pharmaceutical Research, 2020, Vol. 12, Supplementary Issue 2, pp. 1147-1151.

36. Yu.V. Ioni. Effect of Ultrasonic Treatment on Properties of Aqueous Dispersions of Inorganic and Organic Particles in Presence of Water-Soluble Polymers, International Journal of Pharmaceutical Research, 2020, Vol. 12, Issue 4, pp. 3440-3442.

37. N.A. Bulychev, A.V. Ivanov. Study of Nanostructure of Polymer Adsorption Layers on the Particles Surface of Titanium Dioxide, International Journal of Nanotechnology, 2019, Vol. 16, Nos. 6/7/8/9/10, pp. $356-365$.

38. O.A. Butusova. Vinyl Ether Copolymers as Stabilizers of Carbon Black Suspensions, International Journal of Pharmaceutical Research, 2020, Vol. 12, Supplementary Issue 2, pp. 1152-1155.

39. Zaripov R.N., Murakaev I.M., Novikov S.V., Ryapukhin A.V. Corporate Structure for Innovative Enterprises, Russian Engineering Research, 2020,Vol. 40, I. 2, pp. 137-139.

40. BurovaA.Yu.,Ryapukhin A.V.,Muntyan A.R. Reduced hardware costs with software and hardware implementation of digital methods multistage discrete Fourier transform on programmable logic devices, Amazonia Investiga, 2020, Vol. 9, I. 27, pp. 227-233.

41. O.A. Butusova. Adsorption Behaviour of Ethylhydroxyethyl Cellulose on the Surface of Microparticles of Titanium and Ferrous Oxides, International Journal of Pharmaceutical Research, 2020, Vol. 12, Supplementary Issue 2, pp. 1156-1159.

42. Rabinskiy, L.N., Sitnikov, S.A. Development of technologies for obtaining composite material based on silicone binder for its further use in space electric rocket engines// PeriodicoTcheQuimica, 2018, 15(Special Issue 1), p. 390-395. 